\title{
Study on Electroless Nickel Plating Technique at Low Temperature Based on Ultrasonic Wave
}

\author{
Luo Le $\mathbf{L}^{1}$ \\ ${ }^{1}$ Chongqing Vocational Institute Engineering, Chongqing, China,402260 \\ ${ }^{a}$ email
}

Keywords: Electroless Nickel Plating, Low Temperature, Ultrasonic Wave

\begin{abstract}
In order to decrease the plating temperature of electroless nickel plating, this paper introduces the ultrasonic wave into the electroless nickel plating process. First, we give the concept of ultrasonic low-temperature electroless nickel plating, then point out the experimental materials and methods, and finally explore the experimental results from the perspectives of influence of ligand, complex agent, stabilizer and $\mathrm{PH}$ value to provide reference for the study of the low temperature electroless nickel plating.
\end{abstract}

\section{Introduction}

Electroless nickel plating has excellent corrosion resistance, wear resistance and high hardness. It has been widely used in petrochemical, machinery, automobile, electronics, textile and other industries. Commonly, it is used in high temperature environment in domestic and foreign chemical nickel plating process. The process energy consumption, tank wall serious self-deposition, in the plating process of moisture and volatile, plating solution stability difference, low utilization rate of sodium hypophosphite, at high temperature for some materials applied plating will cause deformation and degeneration of the matrix, in addition acidic bath to the tank body material and filter resistance requirements of high temperature high and its application restricted. Ultrasonic electroless nickel plating is changing the mode of energy input, due to low temperature, greatly reduce the energy consumption and applicable to metal with low melting point and easy deformation of plastics and other non-metallic materials. Therefore, the development of low temperature ultrasonic electroless plating has important significance in both industrial applications, or in the chemical plating theory research.

The reason the ultrasonic wave can increase the chemical reaction rate lies in the ultrasonic cavitation. The chemical reaction can occur in the cavitation bubble. At the same time, the impact of ultrasonic cavitation wave damages the metal specimen surface insoluble dirt, the fatigue damage and detachment from the surface dispersed in solution, thereby cleaning the surface of the specimen, the new surface is exposed to increase the reaction surface area to improve the reaction speed. The effect of cavitation on the acceleration reaction is important, but the input of ultrasonic energy increases the internal energy of the reactant and increases the average velocity of the molecule. It is one of the important factors of ultrasonic catalysis to replace the original thermal activation with ultrasonic activation. In the process of acoustic cavitation, the plating solution will produce micro zone high temperature, high pressure, temperature gradient and the pressure gradient, and these phenomena can promote chemical reactions, so the plating liquid temperature by the above phenomenon is overcome and improve not rise too high. When we emphasize the ultrasonic 
cavitation is sound chemical reaction of the active force, we must also pay attention to the contribution of ultrasonic mechanical effect and heat effect of chemical reaction.

\section{Materials and Methods}

Materials. We use the hypophosphite as reducing agent of the plating solution. The acidic plating solution has strong adhesion, high hardness, wear resistance and good corrosion resistance. However, the plating temperature is as high as 85 degrees centigrade. The alkaline bath obtained coating binding force is weak and low phosphorus content in the film, so corrosion resistance and wear resistance are not as good as acidic bath for coating. Acid plating solution is mainly composed of the following components: nickel salt is the main salt of the plating solution of nickel sulfate and nickel chloride. It was found that the deposition rate of $\mathrm{Ni}$ was higher than that of the nickel salt, but the stability of the plating solution decreased. Plating required in the liquid reducing agent and complexing agent dosage is the basis to determine the amount of nickel salt. The concentration of nickel sulfate in the plating solution. In the bath, with the nickel ion is continuously reduced, the lifetime is more and more, it is easy to cause the precipitation of nickel and lead to the decomposition of plating solution. To avoid decomposition and controlling the deposition rate of the bath, plating solution must be added to the complexing agent, complexing agent and nickel from the formation of a stable complex, is used to control the reaction of free nickel ion content, at the same time to inhibit the precipitation of nickel phosphite, plating bath has good stability. The complexing agent used mainly acetic acid, citric acid, amber acid, malic acid and their salts etc.. In order to control the reduction of nickel ions and to make the reduction reaction only on the surface of the substrate, the stabilizer should be added to the plating solution. In addition, coated particles or solid particle in the liquid and the particles may is external impurities into plating solution and plating production of nickel phosphite deposition in liquid, caused by the spontaneous decomposition of the bath. The reason lies in the preferentially adsorbed micelle particles, hindering the reduction of nickel ions.

Methods. We firstly analyse the nano SIC particles after pretreatment on the balance. We put them in to the beaker and add a small amount of deionized water in an ultrasonic generator oscillation. The nano SIC particles can be fully dispersed; PTFE can be directly into the chemical plating liquid. The second phase particles are poured into the chemical composite plating solution, and the second phase particles are fully infiltrated by the bath with a magnetic mixer for at least 1 hours. A beaker of pre plating solution in the constant temperature water bath, heated to a predetermined temperature. A beaker of electroless plating solution into the ultrasonic generator, heated to a predetermined temperature. Using the weight gain method to measure the plating speed, the photoelectric analytical balance for weighing, the plating speed of each point in the curve is the average value of the 3 samples under the same condition. The micro hardness of electroless nickel coating was measured with a digital micro hardness tester. The test load was 25 grams, and the loading time was 5 seconds. The time of alkali cleaning and acid cleaning activation must be in accordance with the regulations. If the time is too long, it will introduce a large error. Plating in the test piece can not be exposed to air to avoid the formation of oxide film on the surface, which will result in decreased binding force.

\section{Result Analysis}

Influence of Ligand. In the high temperature, the use of succinate and malic acid will significantly promote the deposition rate. This experiment is based on the recogonition. We try to 
lower temperature in 60 degrees celsius by changing the energy input method. The investigation in the conventional process and ultrasonic assisted deposition. The concentration of the acid, the concentration of malic acid, the deposition rate and the apparent quality of the samples were observed. Bath composition and process conditions are: nickel sulfate $25 \mathrm{~g} / \mathrm{L}$, sodium sulfate $30 \mathrm{~g} / \mathrm{L}$, butyl acid $10 \mathrm{~g} / \mathrm{L}$, temperature $60 \mathrm{C}, \mathrm{PH} 5$. Under the low temperature and in conjunction with succinic and malic acid, deposition rate is very low which is below the $4 \mu \mathrm{m} / \mathrm{h}$. After the introduction of ultrasonic wave in the plating solution at 60 degrees celsius, the deposition rate is less than that of the conventional electroless plating at the same temperature, but the deposition rate is lower than that at the high temperature. Analysis of the reasons, may be because give the ultrasonic electroless nickel plating to provide energy and provided by heating the heat vary, due to malic acid and nickel ions to form complexes is relatively stable, provided by the ultrasonic energy to overcome the malic acid and other strong ligands on nickel ion bound, difficult to compensate the temperature decrease caused by the activity of nickel ion concentration decreased, thus impeding the deposition reaction speed. When the concentration of malic acid is zero, that is, containing only succinic acid, under the action of ultrasonic assisted chemical plating than conventional electroless deposition rate increased slightly. It may be related with the ligand stability constants.

Influence of Complex Agent. The deposition rate increased with the content of sodium hypophosphite to about $30 \mathrm{~g} / \mathrm{L}$ maximum value, and then decreased. Acidic electroless nickel plating liquid and the increase of sodium hypophosphite concentration has to improve the deposition velocity, but plating liquid sodium hypophosphite concentration is too high, the plating plating tank is easy to occur in the nickel particles, was increased by $\mathrm{N}$ plating of the catalytic core, greatly reduces the stability of plating solution. There are many factors that affect the deposition rate of electroless nickel plating, the quality of the coating and the service life of the plating bath. In addition to the temperature, the concentration of nickel ion and reducing agent, $\mathrm{pH}$ value, complexing agent and stabilizer, etc.. It is generally believed that the choice of the type and concentration of the complexing agent is one of the basic factors to improve the deposition rate and to reduce the temperature of the plating. The stability of the complex is expressed by the stability constant $\mathrm{pK}$, the larger the $\mathrm{pK}$ value, the more stable the complex. When nickel ion and phosphate in the test pieces after the surface redox reaction, if in ordinary constant temperature water bath plating, surface nickel ion and phosphate ion concentration and the bulk solution concentration has a concentration gradient, it can only by diffusion mass transfer process of the specimen surface concentration are added, and in the ultrasonic, due to the forced convection generated by the ultrasonic oscillation, workpiece surface and the bulk solution concentration of almost equal, so greatly reduces the trial and error surface and bulk solution concentration. So the deposition rate of ultrasonic wave is higher than that of conventional electroless plating. The stirring effect and the energy provided by the ultrasonic cavitation are favorable for the diffusion of ions.

Influence of Stabilizer. The effect of power ultrasound can make some immiscible liquid become to good emulsion. This particularly fine emulsion provides a large amount of mutual contact area between the liquid and the liquid, so as to accelerate the chemical reaction between them. The stability of the plating solution was improved obviously under the condition of sulfur pulse stabilizer. In the conventional chemical plating, we added the deposition rate of the sulfur puls. This shows that in the conventional chemical plating, adding sulfur vein can obviously increase the speed of deposition. It is generally believed that the reason for the deposition of nickel in the accelerated electroless nickel plating is that it has a strong tendency to accelerate the electron exchange on the surface of the metal. Deposition process is controlled by surface active, thiourea can penetrate into the surface of the electrode, barrier film role, even dissolve surface film layer, 
thereby increasing the activity of nickel ion; in ultrasonic electroless plating, after the addition of thiourea, plating solution stability enhanced, but deposition velocity variation is not obvious. This is a question that is worth discussing. Analysis may be due to the ultrasonic oscillation, the sulfur veins in specimen surface adsorption capacity decreased, thus weakening the its activating effects of nickel ions, the sulfur veins in ultrasonic electroless plating only to play a stabilizing role, and the deposition rate did not change significantly. When the concentration of sulfur is more than $2.0 m g / L$, whether it is ultrasonic chemical plating, or conventional chemical plating, deposition rate is greatly decreased, this is because when its concentration exceeds a certain value, and even make the whole reaction completely stopped. This is due to the large concentration of the stabilizer.

Influence of PH Value. The experimental results of different concentrations of sodium acetate have different effects on the deposition rate. Sodium acetate addition added to the plating liquid can stabilize the $\mathrm{PH}$ value of the role. The mass concentration increased, enhancing buffer ability. However, the $\mathrm{PH}$ value is too high. It is not conducive to the reaction. With the increase of the concentration of sodium acetate, the percentage of weight gain and nickel deposition rate increased, and the best in $20 \mathrm{~g} / \mathrm{L}$, then increased and decreased. The $\mathrm{PH}$ value of the plating solution has a significant influence on the deposition rate. According to the theory of electroless nickel plating, the nickel ion in the plating solution was reduced to metal nickel under the action of the second phosphate. The reduction ability of sodium phosphate was enhanced with the increase of $\mathrm{pH}$ value of the plating solution. But when the $\mathrm{pH}$ value is too high, in the case of complexing agent concentration is not too high, will likely appear two tendencies: one is the cause of the plating liquid phosphite nickel and nickel hydroxide solubility is greatly reduce the plating solution appear muddy, the second is lead to bath the spontaneous decomposition. On the contrary, if the PH value of the plating solution is too low, the stability of the plating solution will increases and the growth rate of the coating decreases. Therefore, the $\mathrm{PH}$ value of the solution is about 5.75.

\section{Conclusion}

The effect of ultrasonic wave on the deposition rate of electroless nickel plating is related to the stability constant of the nickel ion ligand in the bath. Unlike the conventional electroless nickel plating, the deposition rate of electroless nickel plating is not significantly affected by the stabilizer thiourea. However, the relatively low speed is the bottleneck of low temperature electroless nickel plating process. The problem needs further improvement by scientific researchers.

\section{References}

[1] Liu Junbo, Journal of Weifang College, Vol. 15 (2000) No 1, p.36-38

[2] Chen Hu, Hu Shouliang, Li Jian, Materials Protection, Vol. 47 (2014) No 8, p.15-17

[3] Zhong Huimei, Huang Lizhu, Chen Riyao, Zheng Xi, Chen Zhen, Journal of Fujian Normal University ( Natural Science Edition), Vol. 22 (2006) No 2, p.56-59

[4] Gao Shuxuan, Liu Guichang, Zhao Yu, Materials Protection, Vol. 37 (2004) No 3, p.56-59 